\section{Gastric leiomyoma in a free-living Atlantic bottlenosed dolphin (Tursiops truncatus)}

\author{
D. S. RotsteIn, C. A. HARMS, \\ G. N. LOVEWELL, A. A. HOHN
}

NEOPLASMS in free-living cetaceans are not commonly found, which may reflect the difficulty of assessing animals that usually die in the aquatic environment, negating any postmortem examination or resulting in an advanced state of autolysis. Individual or, occasionally, clusters of neoplasms such as fibroleiomyomas in Beluga whales (Mikaelian and others 2000) have been reported. Tumour types include epithelial, mesenchymal and, less commonly, neuroendocrine tumours (Gulland and others 2001). Renal adenoma, reticuloendotheliosis, lymphosarcoma, squamous cell carcinoma and pancreatic adenoma have been reported in Atlantic bottlenosed dolphins (Tursiops truncatus) (Gulland and others 2001). Mesenchymal tumours are less common in the species; leiomyomas of the digestive tract have been recorded only in single individuals, including in the intestine of an Atlantic white-sided dolphin (Lagenorhynchus acutus) (Geraci and others 1987) and in the stomach of a common dolphin (Delphinus delphis) (Cowan and others 1986). This short communication details the gross, histological and immunohistochemical findings of a gastric leiomyoma in an Atlantic bottlenosed dolphin.

An emaciated, adult, male Atlantic bottlenosed dolphin, $268 \mathrm{~cm}$ in length and weighing $262 \cdot 2 \mathrm{~kg}$, was stranded dead off the coast of North Carolina, USA. Postmortem examination revealed lesions in the digestive, musculoskeletal, urogenital and endocrine systems. Specific gross findings were left mandibular swelling, urinary calculi in the penile urethra, focal proliferations on the penis, adrenocortical cysts, serous atrophy, a healed costal fracture and gastric trematodiasis. In the pylorus, there was a focal intramural mass measuring $3.0 \mathrm{~cm} \times 2.0 \mathrm{~cm} \times 2.0 \mathrm{~cm}$, which was ulcerated on the mucosal surface, although luminal circumference was not reduced (Fig 1). On the cut surface, the mass was firm and white. Gastric contents were absent save for a bilious fluid. Representative sections were collected in 10 per cent formalin and processed routinely.

Histologically, lesions were found in the cardiovascular, respiratory, digestive, endocrine, musculoskeletal and urogenital tracts. Specific microscopic findings unrelated to the pyloric mass included myocarditis, aortitis, pulmonary oedema, gastric trematodiasis (Braunina cordiformis), colitis, hepatic haemosiderosis, proliferative balanitis, urethritis, focal mandibular callus (present before fracture) and adrenocortical cysts.

The pyloric mass was composed of plump spindyloid cells forming interlacing bundles (Fig 2a). Cells had abundant eosinophilic cytoplasm and small, elongated nuclei, with minimal anisocytosis and anisokaryosis. Mitoses were not observed. Moderate inflammation was evident, associated with plasma cells and macrophages.

Based upon the histological findings, two differential diagnoses for the neoplasm were proposed: leiomyoma and gastrointestinal stromal tumour. Immunohistochemical stains were applied to identify the tumours, including the myogenic markers smooth muscle actin (SMA), muscle-specific actin (MSA) and desmin (Des), as well as cytokine markers (c-kit) and neurogenic markers (S-100). Results of staining indicated positive intracytoplasmic staining for SMA (Fig $2 b$ ) and

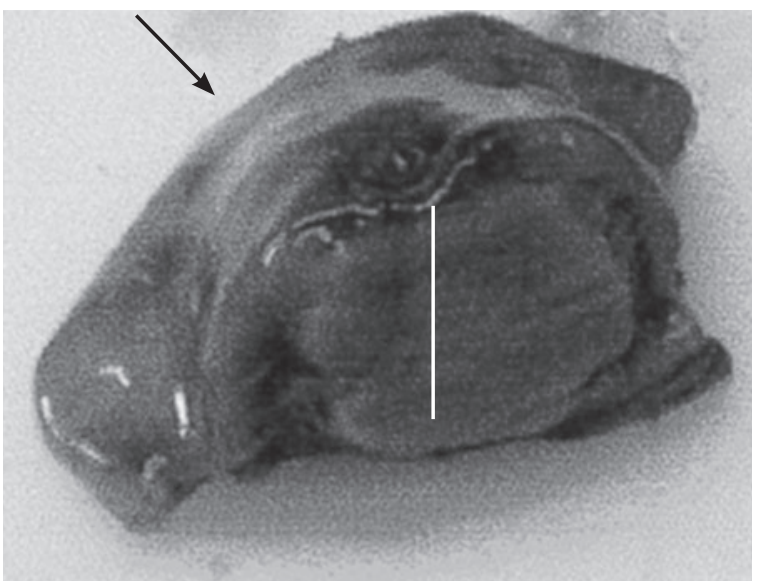

FIG 1: Tumour found in the pylorus of an Atlantic bottlenosed dolphin (Tursiops truncatus) in which the tunica media is expanded by a well-demarcated nodule (white line); the arrow indicates the gastric mucosa

MSA (Fig 2c), rare positive intracytoplasmic staining for c-kit and S-100, and negative staining for desmin. A leiomyoma was therefore diagnosed.

Leiomyomas, which are of smooth muscle origin, exhibit positive immunohistochemical staining for SMA, MSA and desmin; however, in one study of dogs, only 62 per cent of the tumours were positive for desmin and 97 per cent were positive for SMA (Frost and others 2003). Gastrointestinal stromal tumours are proposed to arise from the interstitial cells of Cajal and exhibit positive staining for c-kit, a protooncogene that encodes for a transmembrane tyrosine kinase. Infrequent staining is observed with SMA, MSA and S-100 (Frost and others 2003). Gastrointestinal stromal tumours have been reported in human beings, dogs and horses.

The gastric leiomyoma in this case was an incidental finding; the animal is likely to have died from the combination
Veterinary Record (2007) $160,130-131$

D. S. Rotstein, DVM, MPVM, DACVP, Department of Pathobiology, College of Veterinary Medicine, University of Tennessee, Knoxville, TN 37996-4542, USA

C. A. Harms, DVM, PhD, DACZM,

Department of

Clinical Sciences and Environmental Medicine Consortium, College of Veterinary Medicine, Center for Marine Sciences and Technology, North Carolina State University, Morehead City, NC 28557, USA

G. N. Lovewell,

\section{A. A. Hohn, $\mathrm{PhD}$,}

National Marine Fisheries Service, NOAA Beaufort Laboratory, 101 Pivers Island Road, Beaufort, NC 28516, USA
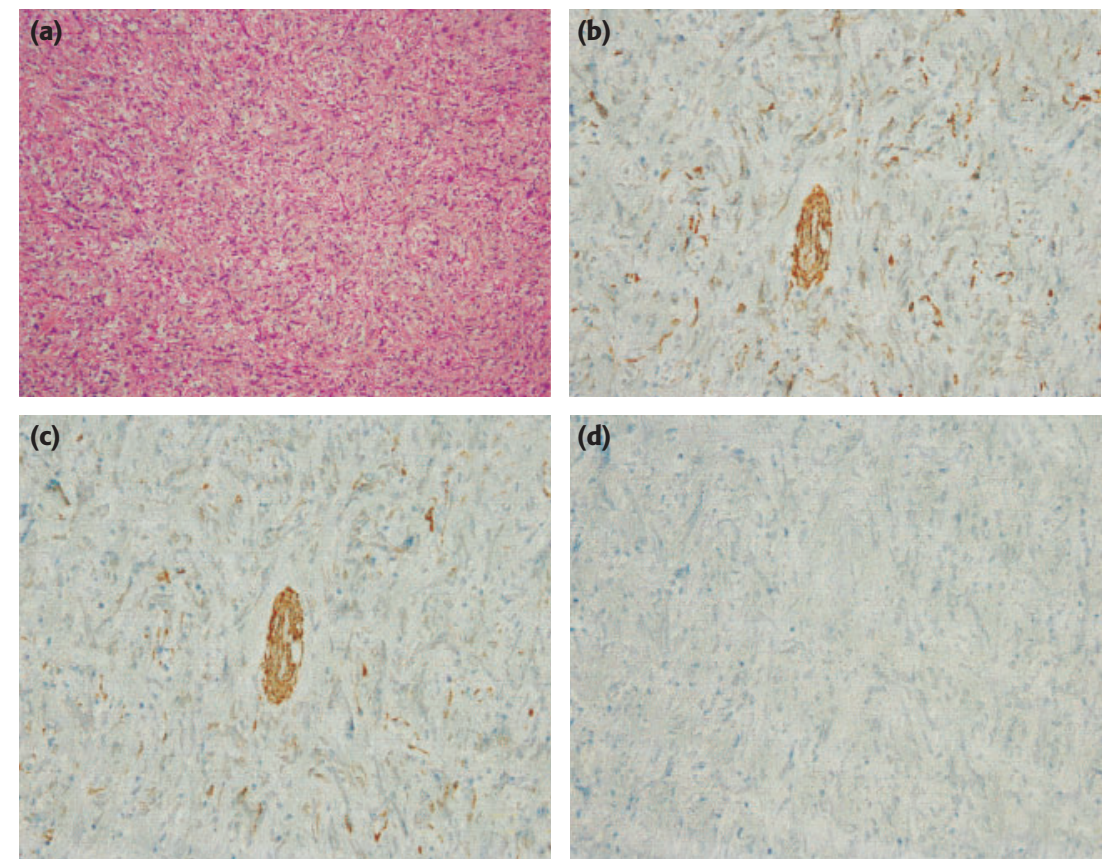

FIG 2: Pyloric mass in an Atlantic bottlenosed dolphin (Tursiops truncatus) in which (a) the gastric tunica muscularis is composed of bundles and fasicles of spindyloid cells. The tumour positively stained for (b) smooth muscle actin and (c) muscle-specific actin, showing intracytoplasmic granules. (d) The vascular tunica media is an internal positive control; there is no background staining of the negative control 
of the mandibular fracture, resulting in inanition, and myocarditis affecting cardiac function. The neoplasma formed a localised mass, resulting in bulging and superficial ulceration of the gastric mucosa. The mass was not associated with the trematodes that were present in the main compartment of the stomach.

Continued reporting and classification of neoplasms in cetaceans and other aquatic animals is an important method of assessing population health. It is from these recorded neoplasms that patterns may begin to emerge, and the impact on the population of individual species can be determined.

\section{References}

COWAN, D. F., WALKER, W. A. \& BROWNELL Jr, R. L. (1986) Pathology of small cetaceans stranded along the southern California beaches. In Research on Dolphins. Eds M. M. Bryden, R. Harrison. Oxford, Oxford University Press. pp 323-367

FROST, D., LASOTA, J. \& MIETTINEN, M. (2003) Gastrointestinal stromal tumors and leiomyomas of the dog: a histopathologic, immunohistochemi$\mathrm{cal}$, and molecular genetic study of 50 cases. Veterinary Pathology 40, 4254

GERACI, J. R., PALMER, C. \& ST AUBIN, D. J. (1987) Tumors in cetaceans: analysis and new findings. Canadian Journal of Fisheries and Aquatic Sciences 44, 1289-1300

GULLAND, F.M.D., LOWENSTINE, L. J. \& SPRAKER, T. (2001) Noninfectious diseases. In CRC Marine Mammal Medicine Handbook. 2nd edn. Eds L. A. Dierauf, F. M. D. Gulland. Boca Raton, Florida, CRC Press. pp 522528

MIKAELIAN, I., LABELLE, P., DORE, M. \& MARTINEAU, D. (2000) Fibroleiomyomas of the tubular genitalia in female Beluga whales. Journal of Veterinary Diagnostic Investigation 12,371-374 